\title{
Innervation and Vasculature of Human Sweat Glands: An Immunohistochemistry-Laser Scanning Confocal Fluorescence Microscopy Study
}

\author{
William R. Kennedy,' Gwen Wendelschafer-Crabb,' and T. Clark Brelje ${ }^{2}$ \\ Departments of ${ }^{~ N e u r o l o g y, ~}{ }^{2}$ Cell Biology and Neuroanatomy, University of Minnesota, Minneapolis, Minnesota 55455
}

\begin{abstract}
Secreting tubules, nerves fibers, and blood vessels in human sweat glands (SGs) were fluorescently stained by immunohistochemical and lectin methods for examination with a laser scanning confocal microscope (LSCM). Using these techniques, the three-dimensional distribution of up to three substances within a single specimen was investigated by collecting a series of optical sections for each of three fluorophores. Each SG received several nerve fibers. These branched into delicate bands of one or more axons that ran longitudinal to the sweat tubule then encircled the tubule. A heavy complement of capillaries was interwoven among the sweat tubules. Sweat ducts were accompanied from the SG toward the skin surface by one or two longitudinally oriented nerve fibers and capillaries. Immunoreactive staining of nerves was heaviest with protein gene product 9.5 antibody, but triple labeling showed that immunoreactivity to calcitonin gene-related peptide, vasoactive intestinal polypeptide, and synaptophysin was also present in the same axons. Substance P-immunoreactive axons were sparse in SGs but were present in other areas of the skin. The techniques used have considerable potential in examination of human skin biopsies for diagnosis of disorders affecting the somatic and autonomic nervous systems.
\end{abstract}

[Key words: blood vessels, confocal microscopy, immunohistochemistry, nerves, PGP 9.5, skin biopsy, sweat gland, sudomotor]

Full description of the normal innervation of human sweat glands (SGs) and recognition of changes produced by disease have previously been handicapped by failure of the classical staining methods to stain consistently all nerves within the glands. With the development of immunohistochemical methods, it has become possible to visualize sudomotor nerves clearly and to determine their neuropeptide content (Dalsgaard et al., 1989b; Karanth ct al., 1989, 1991). However, the convoluted folding of sweat tubules and intertwining of sudomotor axons have limited previous descriptions to the observation that there are a large number of nerve fibers around and between sweat tubules. The advent of the laser scanning confocal microscope (LSCM) made it possible to provide a more detailed description

\footnotetext{
Received Apr. 5, 1994; accepted May 5, 1994.

This work was funded by grants from the National Institutes of Health (NS 26348, NS 31397) and Toray Industries Inc. We thank Michele Illies and Daniel Rupperman for their diligent technical assistance.

Correspondence should be addressed to William R. Kennedy, M.D., Box 187 UMHC, 420 SE Delaware Street, Minneapolis, MN 55455.

Copyright (C) 1994 Society for Neuroscience $0270-6474 / 94 / 146825-09 \$ 05.00 / 0$
}

of nerves, capillaries, and sweat tubule of intact SGs. The rejection of out-of-focus light by this instrument allows the acquisition of optical sections at $2-5 \mu \mathrm{m}$ intervals throughout the depth of very thick sections of the specimen by adjusting the position of the focal plane. The series of digitized optical images that are acquired can be used to form high-contrast three-dimensional reconstructions for examination of the interrelationships between stained structures. We have used the combination of immunohistochemistry and LSCM to provide a detailed description of the innervation of human epidermis, a skin layer in which nerve fibers had previously been difficult to study (Kennedy and Crabb-Wendelschafer, 1993). This report extends our observations to the three-dimensional organization of nerves, blood vessels, secreting tubules, and sweat ducts of human SGs. This approach demonstrates the potential for skin biopsy to be used as a method for diagnosis of disorders affecting the somatic sensory and autonomic nervous systems.

\section{Materials and Methods}

Full thickness skin biopsies were taken with informed consent from the incision site (abdomen, jaw, back, arm, or toe) of normal volunteers at the time of surgery. Subjects included males and females aged 11 to 65 years. The project was approved by the University of Minnesota Committee for Use of Humans in Research.

Skin samples were immediately fixed in $4^{\circ} \mathrm{C}$ Zamboni's fixative (1967) and held at $4^{\circ} \mathrm{C}$ overnight. They were transferred to $20 \%$ sucrose phosphate-buffered saline (PBS) and refrigerated for $24 \mathrm{hr}$ or until sectioned. Sections $(100 \mu \mathrm{m})$ were cut with a freezing, sliding microtome (American Optical Co., Buffalo, NY), placed in spot plates, and flooded with 0.1 M PBS with $0.3 \%$ Triton X-100 (Sigma, St. Louis, MO) and $1 \%$ normal goat or donkey serum (PBS-TS). Immunohistochemical staining was performed with the antibodies listed in Table 1 . Nonimmune sera was used for negative controls. Antibodies were visualized with cyanine 3.18 or cyanine 5.18 fluorophores conjugated to appropriate goat or donkey secondary antibodies (Jackson Immunoresearch, West Grove, PA). Secondary antibodies were routinely used at a 1:200 dilution. Sections were also treated with $50 \mu \mathrm{g} / \mathrm{ml}$ Ulex europaeus agglutinin (UF.A I) labeled with fluorescein isothiocyanate (Vector, Burlingame, CA) to stain blood vessels, SG tubules, and hair follicles (Holthofer et al., 1982). Floating sections were incubated with each antibody overnight at $4^{\circ} \mathrm{C}$ on a rotating table. Samples were washed in four changes of PBS-TS for $4 \mathrm{hr}$ between subsequent incubations. Washed sections were dried onto gelatin-coated coverslips, dehydrated through alcohols, cleared with methyl salicylate (Fisher), and mounted in DPX (Fluka, Ronkonkoma, NY).

Specimens were initially examined with a Nikon Microphot SA epifluorescence microscope (Melville, NY) to select areas for further examination in an MRC-600 Confocal Imaging System equipped with a krypton/argon ion laser (Bio-Rad Life Science, Hercules, CA). Triplelabeled sections were imaged using three filters appropriate for the specific visualization of fluorescein, cyanine 3.18 , and cyanine 5.18 (Brelje et al., 1993). Typically, a series of images of optical sections (i.e., a Z-series) was acquired at $2-4 \mu \mathrm{m}$ intervals throughout the depth of the 
Table 1. Antisera used in immunohistochemistry

\begin{tabular}{llll} 
Antibody to & Abbreviation & Dilution & Source \\
\hline Protein Gene Product 9.5 (mouse monoclonal) & m-PGP 9.5 & $1: 800$ & Ultraclone, Wellow, UK \\
Protein gene product 9.5 (rabbit polyclonal) & r-PGP 9.5 & $1: 800$ & Ultraclone, Wellow, UK \\
Substancc P (rabbit polyclonal) & SP & $1: 1000$ & Incstar, Stillwater, MN \\
Calcitonin gene-related peptide (rabbit polyclonal) & CGRP & $1: 1000$ & Amersham, Arlington Heights, IL \\
Vasoactive intestinal protein (rabbit polyclonal) & VIP & $1: 1000$ & Incstar, Stillwater, MN \\
Synaptophysin (mouse monoclonal) & Synap & $1: 100$ & Boehringer Mannheim, Indianapolis, IN \\
Keratin 8, 18, 19 (mouse monoclonal) & Ker8 & $1: 400$ & BioGenex, San Ramon, CA \\
Type IV collagen (mouse monoclonal) & Col-IV & $1: 800$ & Chemicon, Temecula, CA \\
\hline
\end{tabular}

specimen. Each image could be viewed individually or the entire series could be projected into a single in-focus image using software supplied with the microscope. The visibility of lightly stained fibers in the projections could be increased by enhancing the contrast of individual images with computer processing. Stereo pairs were prepared by forming two projections at different viewing angles from the same Z-series. The final images were printed with a Kodak ColorEase thermal dye diffusion printer (Rochester, NY).

\section{Results}

Images collected on the LSCM at low magnification depicted the general pattern of fluorescently stained structures in skin. Dermal nerve bundles were best localized by antibodies to protein gene product 9.5 (PGP 9.5). During their course in the dermis nerve bundles divided to innervate $\mathrm{SGs}$, arrector pili muscles, hair follicles, arterioles, and the epidermis (Fig. 1). Myelinated nerves fibers were chiefly in the large nerve trunks with occasional single fibers approaching the subepidermis. Myelinated fibers in a nerve bundle often passed close to an $\mathrm{SG}$, but the unmyelinated axons entering the SG did not arise from myelinated nerve fibers and the axons enwrapping the sweat tubule were not immunoreactive for antibody to myelin basic protein (MBP). Nerve bundles and single nerve fibers in the dermis were always associated with capillaries; in fact, it was unusual to find dermal capillaries that were not associated with nerve fibers.

SGs were located in the reticular dermis. In hairy skin they were often associated with hair follicles at a level near the bulge region, below the sebaceous glands. SG secretory tubules and their convolutions were most clearly visualized when their associated basement membrane was localized using antibody to type IV collagen (Col-IV) (Fig. 2). Col-IV immunoreactivity (ir) was also useful for staining basement membrane of follicular structures (including sebaceous glands), the epidermal/dermal border, and blood vessels.

The association of two antigens in the same tissue was assessed by double staining with primary antibodies from different species using separate fluorescent labels that were visualized specifically using different filter sets. In Figure 3, mouse anti-ColIV was localized with cyanine 3.18 fluorophore while rabbit anti-PGP 9.5 was localized with cyanine 5.18 fluorophore. The coiled, tightly bundled structure of the secreting tubule of the SGs and the intertwined capillaries were revealed with the ColIV immunolocalization (Fig. $3 A$ ). The axonal wrapping of the sweat tubule was so extensive that the outline of the tubule could be determined by the pattern of immunoreactivity of PGP 9.5 (Fig. 3B).

The relationship between sweat tubules, nerves, and blood vessels in the same sweat glands was examined in triple-labeled specimens. Antibody to cytokeratins 8, 18, and 19 (Ker8) reacted exclusively with the tubules of SGs in human skin (Fig. $4 A$ ) (Yoneda et al., 1991). Nerves localized with PGP 9.5 antibody were present on the sweat tubule and the arteriole entering the sweat gland (Fig. $4 B$ ). UEA I, a lectin that reacts with fucose moieties of the endothelium of blood vessels and follicular structures of the skin, was useful to detect arterioles, venules, and capillaries. In the SG, UEA I was primarily localized on capillaries. A large number of UEA I-reactive capillaries were intertwined with the sweat tubule in Figure $4 C$. The most dense area of the SG capillary network was at the entry of the arteriole into the SG. The arteriole could often be seen adjacent to the SG with its capillary branches extending into the region of the tubules. The arteriole was distinguished by its size, about $30 \mu \mathrm{m}$ (Fig. $4 C$ ), and the presence of several axons woven along its length in a characteristic innervation pattern (Figs. $3 B, 4 B$ ).

The innervation of the SG was derived from one to two nerve bundles that gave rise to multiple sudomotor axons. These divided into the complex array of unmyelinated axons found in association with the SG tubule (Fig. 5). The course of single axons could be followed by viewing the entire Z-series projected in stereo (Fig. 5A), whereas detailed focal information was best obscrved in individual optical sections of the Z-series (Fig. $5 B-$ $D)$. Nerves occasionally formed a small plexus from which several axons emerged to wrap around a portion of the sweat tubule (Figs. $5 A, 6$ ). The most common pattern of SG innervation was the wrapping of sudomotor axons around the secretory tubule so that the tubule was encircled by axons at loosely defined intervals, either singly or in bundles of two to five axons (Fig. 6). Encircling axons occasionally branched but short, abruptly ending branches were unusual. The isolated, terminal end of a tubule viewed at higher magnification in Figure 7 further reveals the intricacy of the axonal wrapping.

SG ducts were accompanied toward the surface of the skin by one or two capillaries with periodic interconnections that sometimes wrapped around the duct (Fig. 8A). The duct was sparsely innervated by one or two axons that coursed with it toward the epidermis closely following the course of the capillaries (Fig. 8B).

Nerves to human SGs were most clearly seen when reacted to antibody to PGP 9.5. They were also immunoreactive in different degrees to calcitonin gene-related peptide (CGRP), vasoactive intestinal peptide (VIP), substance P (SP), and synaptophysin (Synap). The interrelationships between nerves, neuropeptide content, SG structure, and vascularity were assessed by triple staining for UEA I, PGP 9.5, and a selected neuro- 

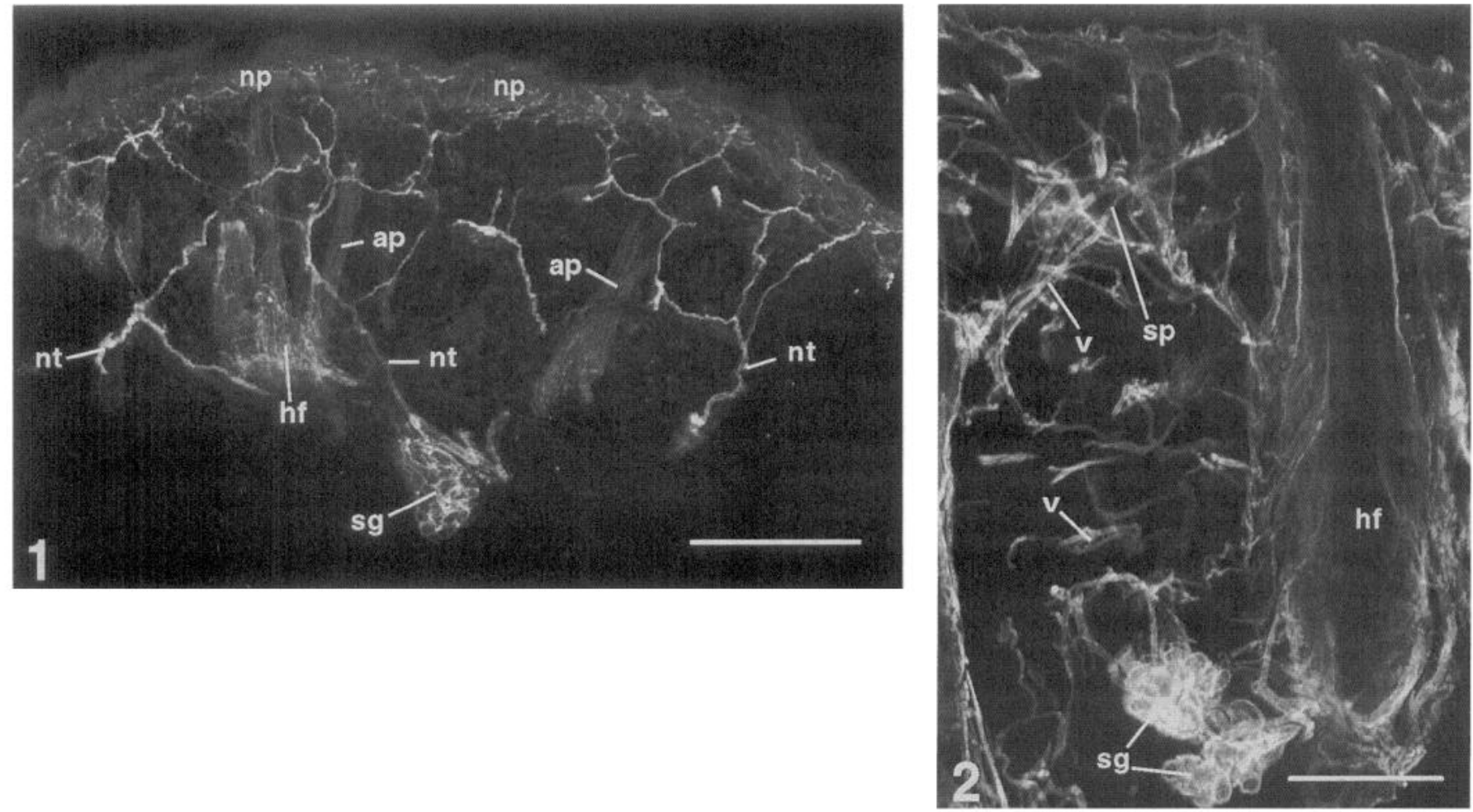

Figure 1. A low-magnification image of nerves in a thick section of human skin. This section was stained with rabbit polyclonal anti-PGP 9.5 and cyanine 3.18-labeled donkey anti-rabbit IgG. A hair follicle $(h f)$, associated SGs $(s g)$, and arrector pilorum muscles $(a p)$ are shown. Large nerve trunks $(n t)$ rise through the dermis to form the subepidermal neural plexus $(n p)$. Epidermal staining is not clearly seen at this magnification. This image is a projection of 21 optical sections taken at $5 \mu \mathrm{m}$ intervals with a $4 \times$ Nikon plan apochromat objective. Scale bar, $500 \mu \mathrm{m}$.

Figure 2. A low-magnification image showing structures outlined by basement membrane staining in a thick section of human skin. This section was stained with mouse monoclonal anti-collagen type IV (Col IV) and cyanine 3.18-labeled goat anti-mouse IgG. A hair follicle ( $h f$ ) is prominent with surrounding capillaries and vessels. An associated SG $(\mathrm{sg})$ is near the base of the follicle. Numerous vessels and capillaries $(v)$ course through the dermis, many entering into the subepidermal plexus $(s p)$. This image is a projection of 26 optical sections taken at $2 \mu \mathrm{m}$ intervals with a $4 \times$ Nikon plan apochromat objective. Scale bar, $500 \mu \mathrm{m}$.
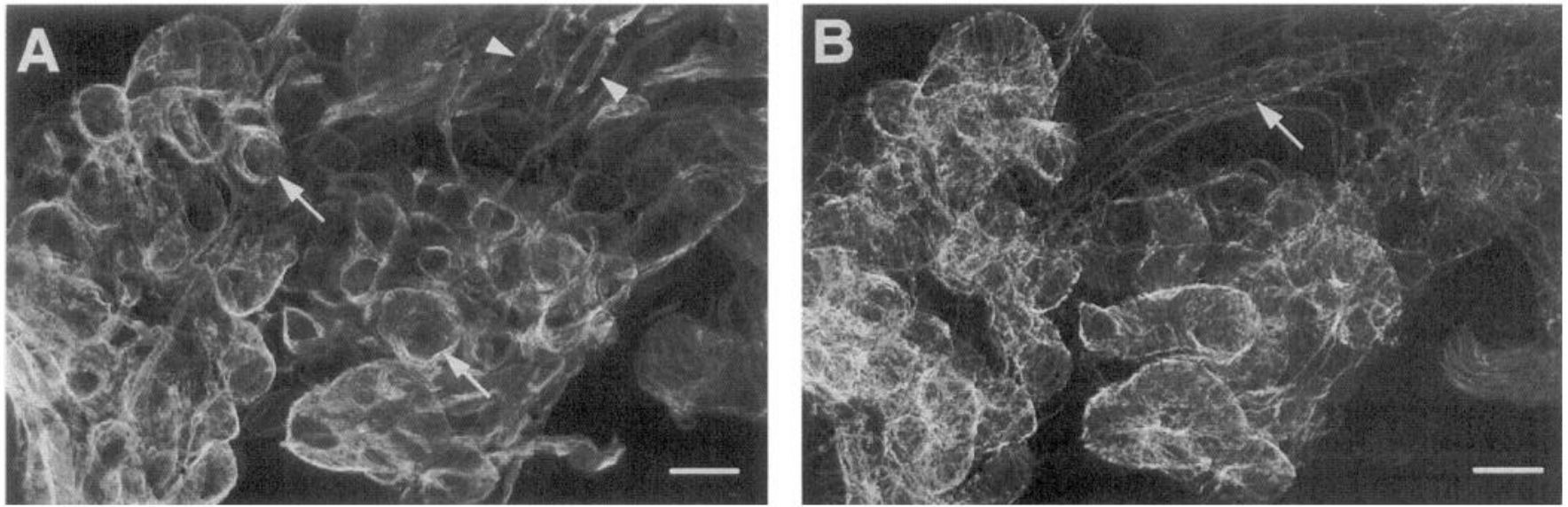

Figure 3. Relationship of basement membrane-delineated sweat tubule and nerves in a SG. This section is double stained with rabbit polyclonal anti-PGP 9.5 and cyanine 3.18-labeled donkey anti-rabbit IgG, and then with mouse monoclonal anti-Col IV and cyanine 5.18-labeled donkey anti-mouse IgG. Two images, each taken from the same site but at different wave lengths, reveal the staining pattern of two distinct antigens in the same tissue. These images are projections of 16 optical sections taken at $4 \mu \mathrm{m}$ intervals with a $10 \times$ Nikon Fluor objective. $A$, Col-IV staining of basement membrane surrounding the sweat tubule reveals the tightly packed coiled nature of the sweat tubule (arrow). Intertwining capillaries $(10 \mu \mathrm{m}$ in diameter) can also be seen (arrowheads). B, Rabbit polyclonal anti-PGP 9.5 immunostaining of nerves on the sweat tubule shown in $A$ reveals an extensive array of axons encircling the convoluted sweat tubule. In some areas, the encircling pattern of axons on the tubule is visible. An artery can be recognized by its characteristic pattern of innervation (arrow). Scale bars, $100 \mu \mathrm{m}$. 

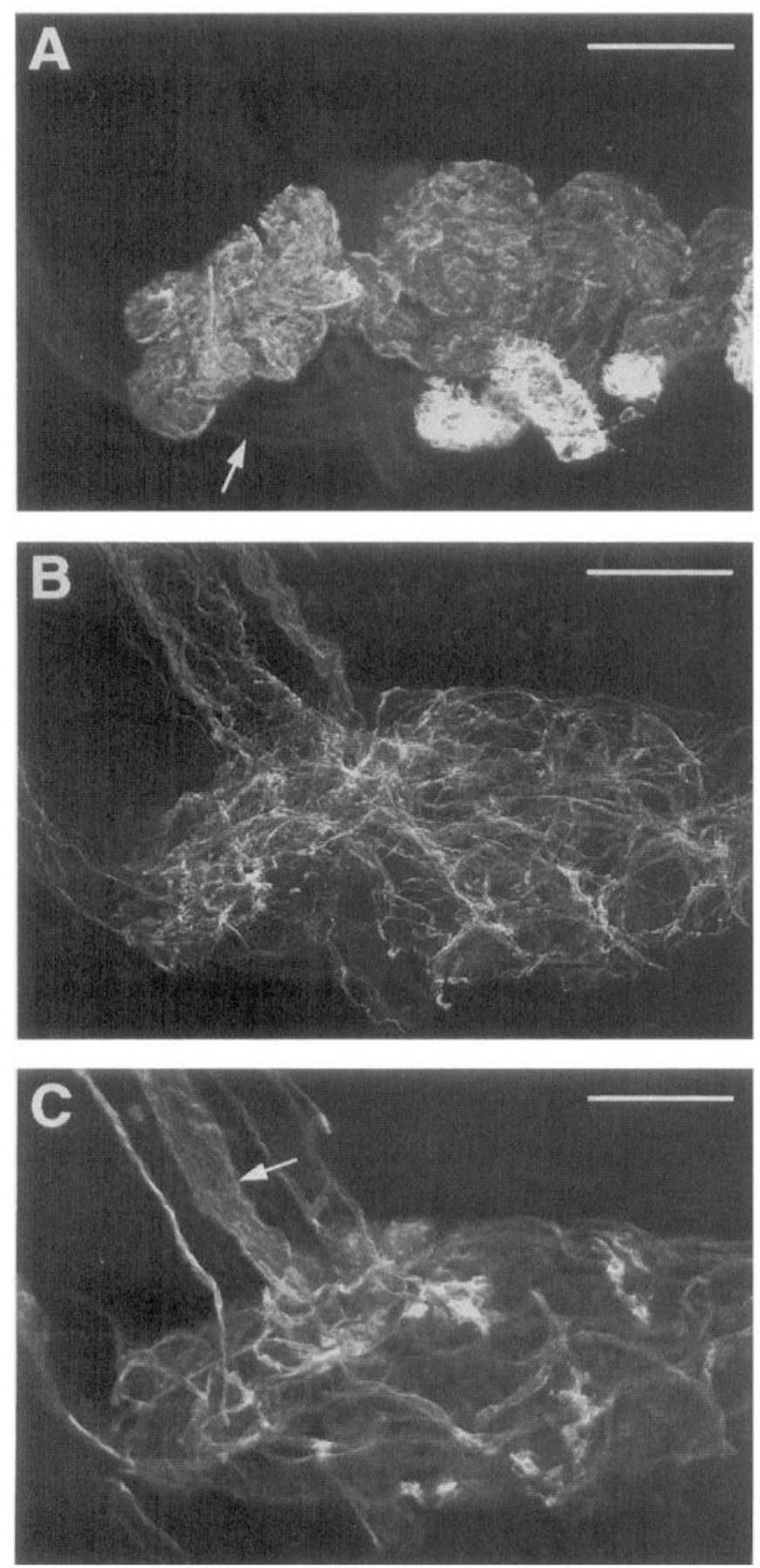

Figure 4. Triple stain of one SG to show tubule structure, innervation, and vasculature. This section was triple stained with mouse monoclonal anti-cytokeratins 8,18 , and 19 and cyanine 5.18-labeled goat antimouse IgG, rabbit polyclonal anti-PGP 9.5 (R-PGP 9.5), and cyanine 3.18-labeled goat anti-rabbit IgG, and then fluorescein-labeled UEA I. These images are projections of 16 optical sections collected at $2 \mu \mathrm{m}$ intervals with a $20 \times$ Nikon Fluor objective. $A$, Antibody to Ker8 was used to stain the tubule of the SG. The staining reveals the complex nature of the tubule. The duct of the sweat gland has minimal reactivity but is visible (arrow). Blood vessels are not immunoreactive to Ker8. $B$, R-PGP 9.5-ir is specific to nerves. It reveals the rich innervation that is characteristic of the SG. The coiling of the nerves around the SG tubule is present but not well seen because of the thickness of the section. Several nerves cover the arteriole (upper left). $C$, UEA I binds to blood vessels and reveals the extensive capillary network that is present in the SG. The large vessel (arrow) entering the SG is an arteriole. Scale bars, $100 \mu \mathrm{m}$. peptide. SP-ir axons were sparse (Fig. 9A). When present, SP colocalized in nerves which also stained for PGP 9.5 (Fig. 9B), but the staining for SP was less intense. Capillaries localized with UEA I in same section were not associated with the SP containing nerves. CGRP-ir was usually present in nerves in SGs (Fig. 10A). Most axons that were reactive for PGP 9.5 were also immunoreactive for CGRP but appeared slightly thicker when stained with PGP 9.5 (Fig. 10B). VIP was also present in the PGP 9.5-ir nerves in SGs (Fig. $11 A, B$ ). Synap-ir axons appeared more bead-like and were present throughout the SG (Fig. $12 A)$. They also colocalized with PGP 9.5-containing neurons (Fig. 12B). Regardless of the method of staining, sudomotor nerves were more closely associated to the sweat tubules than with the capillary network.

\section{Discussion}

Application of immunohistochemical and lectin methods to thick sections of skin resulted in staining of the entire compliment of nerves and blood vessels within sweat glands for examination in the LSCM and epifluorescence microscope. The LSCM performed optical sectioning and collection of in-focus images of selected thickness from desired depths in skin sections of 100 $\mu \mathrm{m}$ or greater. This augmented the collection of data for threedimensional reconstructions of SGs and made it possible to examine their innervation and vasculature. The ability to sample images at desired wavelengths enabled collection of three types of data from the same structures in a specimen.

Nerves to SGs are usually described in general terms, such as being present in large numbers and to closely approach the secretory coils (Vaalasti et al., 1985; Dalsgaard et al., 1989a,b; Karanth et al., 1991). This report extends the observations to the organization of SG innervation, its relationship to sweat tubules and intraglandular capillaries and its immunoreactivity to various neuropeptides. We observed that each SG received multiple nerve fibers. These nerves divided into delicate bands of one or more axons that ran longitudinal to the axis of sweat tubules then branched to encircle tubules at intervals. A heavy complement of capillaries were interwoven among sweat tubules. Sweat ducts were accompanied from the SG toward the skin's surface by one or two longitudinally oriented nerve fibers and one or two capillaries with interconnections. The most intense staining of nerves was observed with antibody to PGP 9.5. Immunoreactivity to VIP, CGRP, and Synap was present in virtually all PGP 9.5-ir axons, but generally neuropeptidestained axons appeared thinner than PGP 9.5-ir axons. In agreement with others (Dalsgaard et al., 1989b; Karanth et al., 1991), we identified few SP-ir nerves in SGs in contrast with the large numbers detected in cat SGs. If large numbers of SP-ir axons had been present they should have been observed because SPir axons were clearly visible in nerve trunks, around larger blood vessels, on hair follicles, and extending into the middle layers of epidermis in the same sections. We could not determine any special relationship between the few SP-ir axons and the arterioles entering the SG or with the secreting tubules. The slender filamentous, sometimes interrupted Synap-ir axons are representative of axon enlargements filled with clear synaptic vesicles (Jahn et al., 1985).

Immunoreactive staining to neuropeptides was described by Lundberg et al. (1979) for VIP in sympathetic neurons to exocrine SGs of cats. It was later demonstrated that some VIPcontaining neurons in sympathetic ganglia also contained CGRP (Landis and Fredieu, 1986) and that VIP and CGRP coexisted 

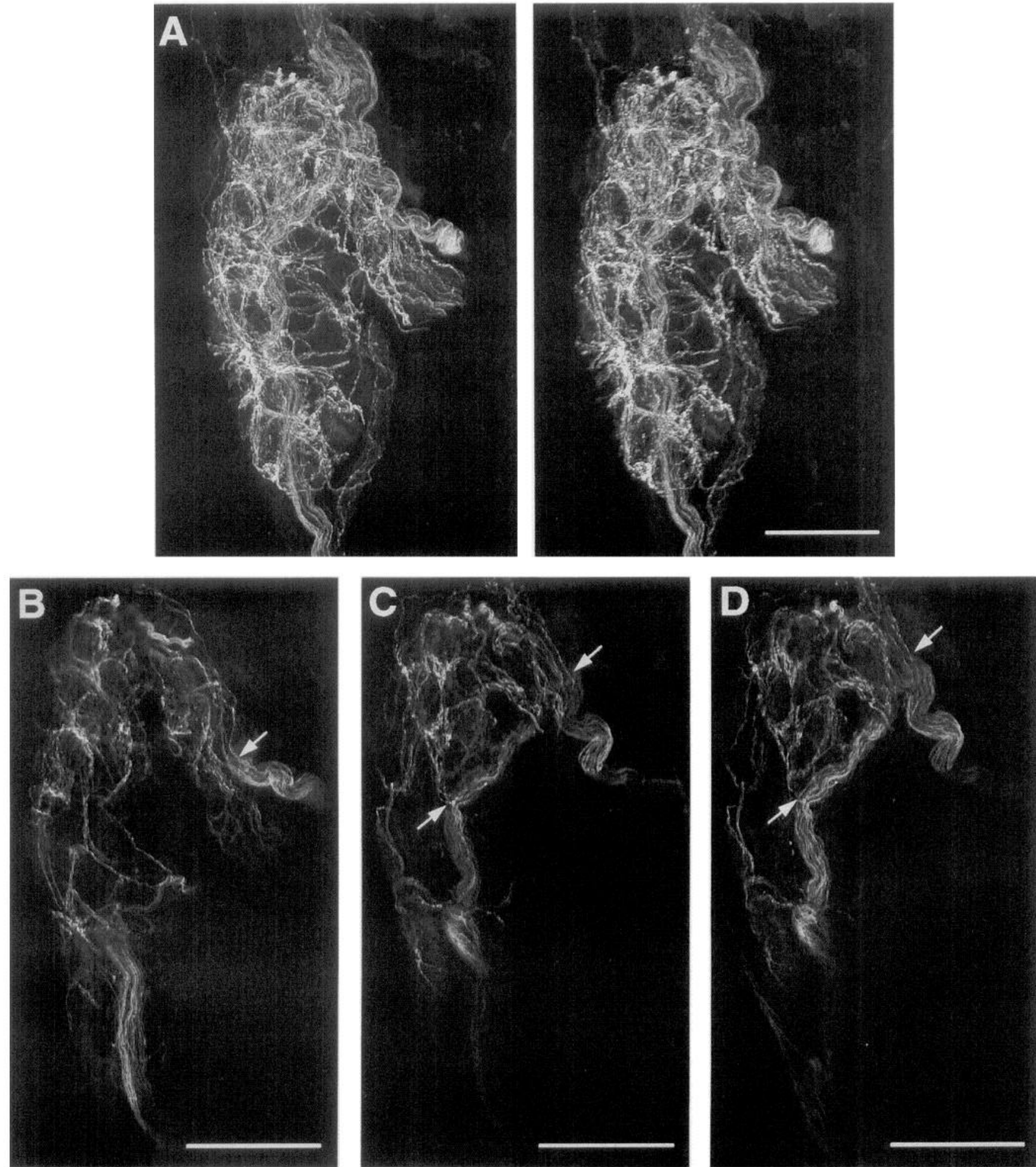

Figure 5. Multiple axons innervating an SG tubule. This section was stained with polyclonal rabbit anti-PGP 9.5 and cyanine 3.18-labeled goat anti-rabbit IgG. The images were collected using a $20 \times$ Nikon Fluor objective. $A$, Stereo pair constructed from a series of optical sections acquired through a sweat gland that receives a large nerve bundle. Multiple axons emerge from the nerve bundle to innervate the sweat gland. The second image is projected with a one pixel shift. Use a stereo viewer to achieve the three dimensional effect. The images are projections of 26 optical sections collected at $1 \mu \mathrm{m}$ intervals. $B-D$, Examination of selected individual optical sections that comprise the above image reveals a number of axons that arise from the nerve bundle to innervate the SG tubule (arrows). Scale bars, $100 \mu \mathrm{m}$. 

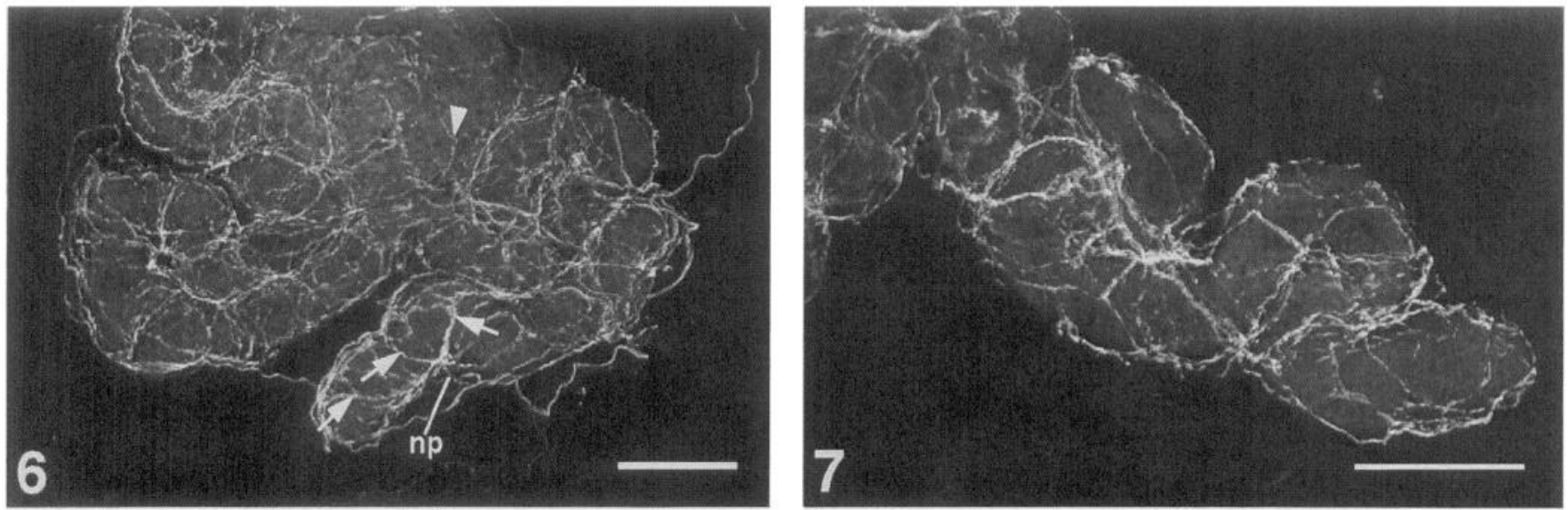

Figure 6. R-PGP 9.5-ir in the SG. This section was stained with polyclonal rabbit anti-PGP 9.5 and cyanine 3.18-labeled goat anti-rabbit IgG. The axons are distinct and the pattern of innervation is apparent. The sweat tubules were made visible by adjusting image contrast to better show the spacial relationship of the axons to the tubules. A plexus of nerves $(n p)$ gives rise to several single axons that wrap around the sweat tubule at loosely defined intervals, either singly or as bundles of two to five axons (small arrows). Encircling axons occasionally branch (arrowhead), but short, abruptly ending branches are unusual. This image is a projection of 21 optical sections collected at $1 \mu \mathrm{m}$ intervals with a $20 \times$ Nikon Fluor objective. Scale bar, $100 \mu \mathrm{m}$.

Figure 7. R-PGP 9.5-ir in the SG. This section was stained with polyclonal rabbit anti-PGP 9.5 and cyanine 3.18-labeled goat anti-rabbit IgG. The intricacy of the axonal wrapping is better discerned in this fortuitously isolated, terminal, blind end of a sweat tubule. This image is a projection of 31 optical sections collected at $1 \mu \mathrm{m}$ intervals with a $20 \times$ Nikon Fluor objective. Scale bar, $100 \mu \mathrm{m}$.
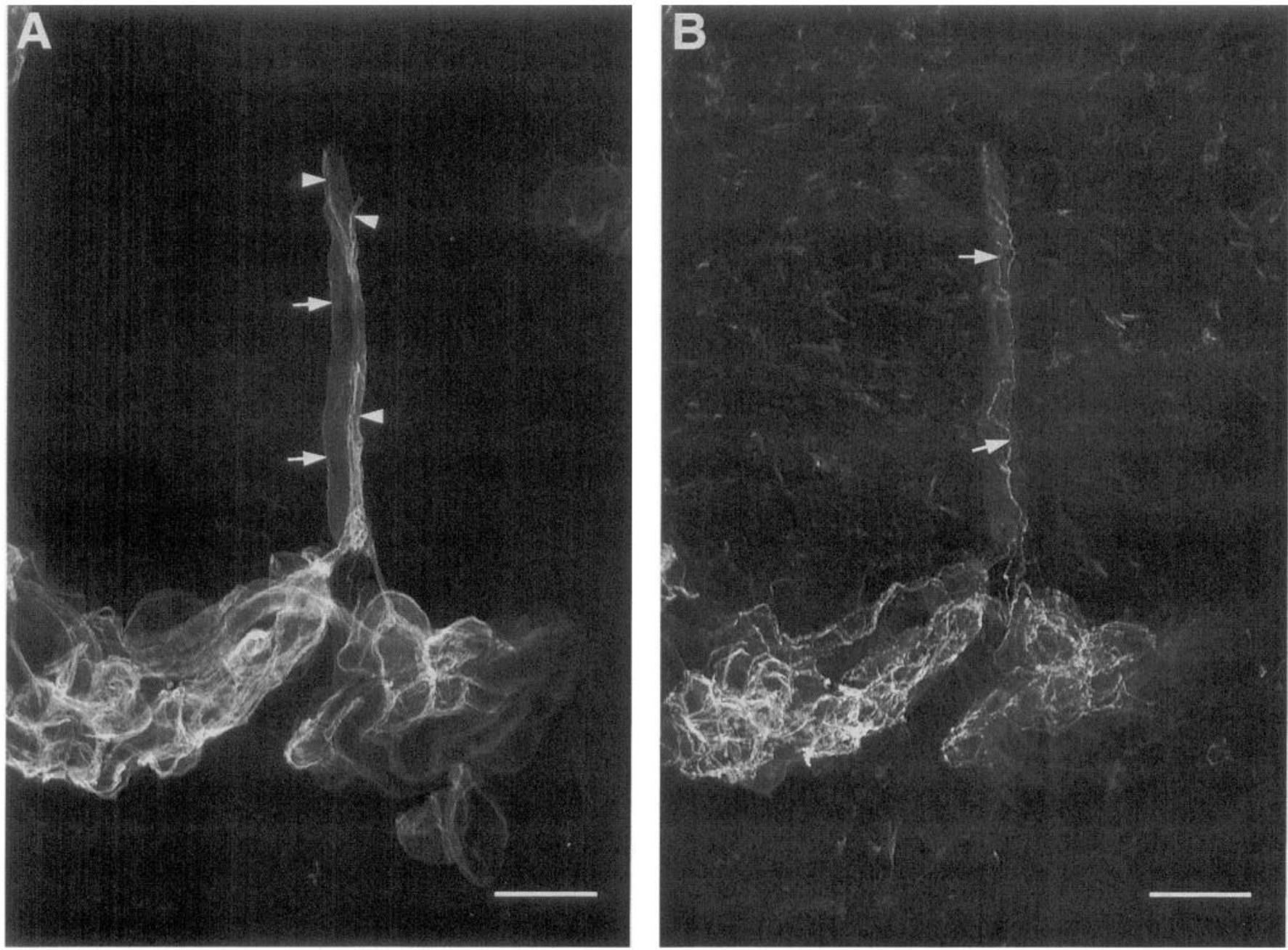

Figure 8. The innervation and vasculature of the SG duct. This section was stained with mouse monoclonal anti-Col IV and cyanine 3.18-labeled goat anti-mouse IgG, and then rabbit polyclonal anti-PGP 9.5 and cyanine 5.18-labeled goat anti-rabbit IgG. These images are projections of 19 optical sections collected at $2 \mu \mathrm{m}$ and taken at different wave lengths from the same area. A $10 \times$ Nikon Fluor objective was used. $A$, The basement membrane immunoreactivity of Col-IV reveals the structure of the sweat tubule and its duct (arrows) and capillaries (arrowheads) parallel to the duct and on the duct. The capillaries frequently wrap around the ascending sweat duct. $B$, Typically, the duct is accompanied to the epidermis by one or two axons (arrow). These axons usually accompany the capillaries. Scale bars, $100 \mu \mathrm{m}$. 

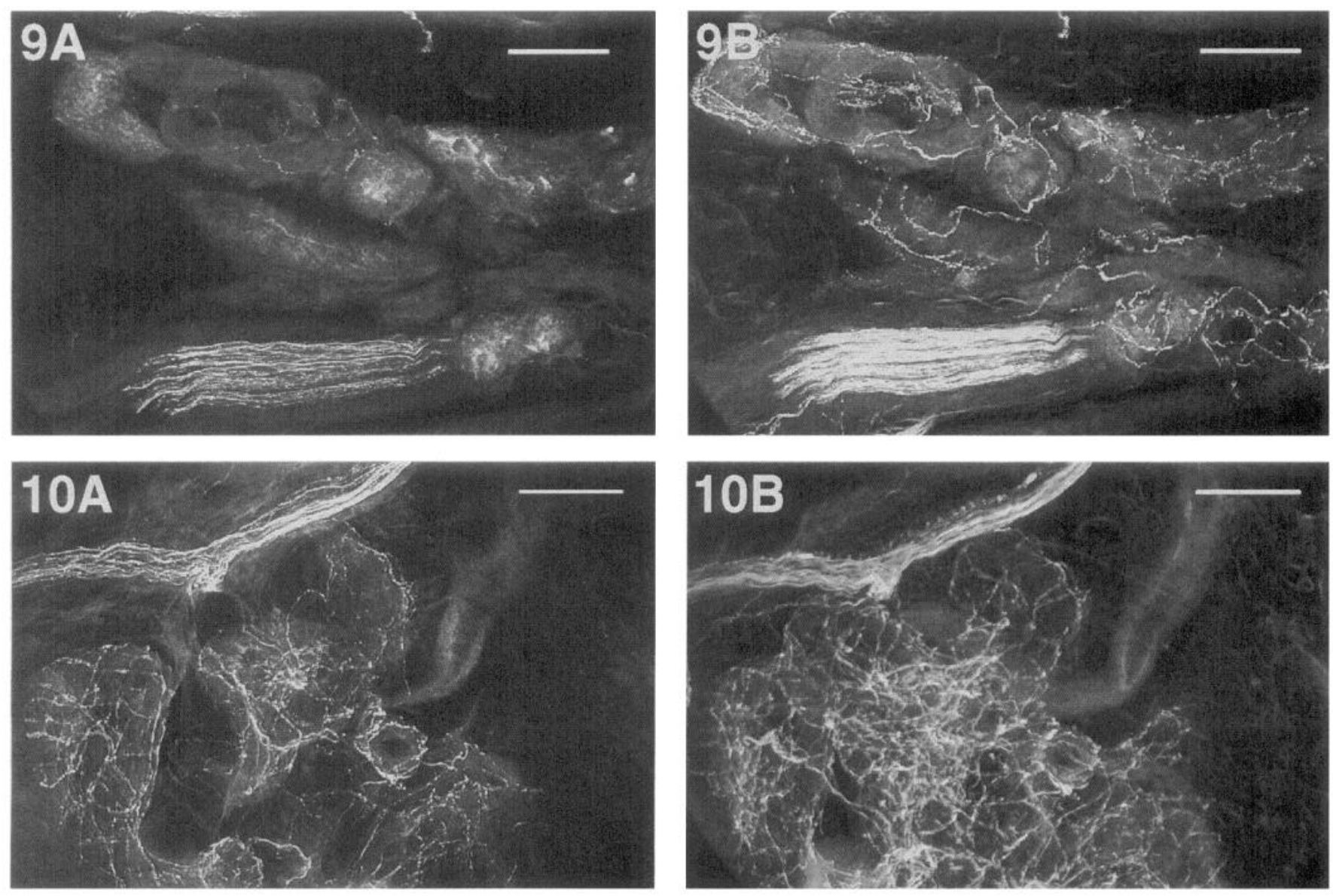

Figure 9. The interrelationship of PGP 9.5-ir and SP-ir in the SG. This section was double stained with polyclonal rabbit anti-SP and cyanine 3.18-labeled goat anti-rabbit IgG, and then monoclonal mouse anti-PGP 9.5 (M-PGP 9.5) and cyanine 5.18-labeled goat anti-mouse IgG. Each set of images was collected from the same SG using different filter sets to visualize the appropriate fluorophore. These micrographs are projections of four optical sections collected at $2 \mu \mathrm{m}$ intervals with a $20 \times$ Nikon Fluor objective. $A$, A small number of SP-containing axons are associated with the tubule of this SG. A large nerve bundle with many SP-containing axons is located below the SG. B, M-PGP 9.5 immunostaining of nerves in the same SG reveals numerous axons coursing along and wrapping around the tubules. The large nerve bundle is heavily stained. Scale bars, $100 \mu \mathrm{m}$.

Figure 10. The interrelationship of m-PGP 9.5-ir and CGRP-ir in the SG. This section was double stained with polyclonal rabbit anti-CGRP and cyanine 3.18-labeled goat anti-rabbit IgG, and then monoclonal mouse anti-PGP 9.5 and cyanine 5.18-labeled goat anti-mouse IgG. The micrographs are projections of four optical sections collected at $2 \mu \mathrm{m}$ intervals with a $20 \times$ Nikon Fluor objective. $A$, Numerous CGRP-containing axons encircle the tubules of the SG. The large nerve bundle also contains CGRP-containing axons. $B$, M-PGP 9.5 localization is seen in essentially the same pattern as seen with CGRP. The two antigens coexist in the same neurons. Scale bars, $100 \mu \mathrm{m}$.

in sympathetic nerves in SGs (Lindh et al., 1987) together with large numbers of SP-ir nerves (Lindh et al., 1988). Most nerves in human SGs are also immunoreactive to VIP (Vaalasti, 1985). The distribution of immunoreactivity to the general neuronal marker PGP 9.5 is of particular interest because of the clarity of staining obtained (Gulbenkian et al., 1987; Wilson et al., 1988; Dalsgaard 1989b; Karanth et al., 1991).

The demonstration that human SGs receive multiple axons complements the physiological evidence for multiple innervation of mouse SGs (Kennedy et al., 1984a). The abundance of neurites with many Synap-ir varices suggests that the several sudomotor axons have numerous synaptic contacts. This is also suggested by the abundance of vesicle-filled sudomotor axons seen in electron micrographs (Quick et al., 1984). We have previously suggested the presence of multiple innervation of SGs from evidence accumulated during nerve stimulation experiments in mice and rats. We postulated that this explained the apparent resistance of SGs to complete denervation in progres- sive polyneuropathy in humans (Kennedy et al., 1984b) and the fast recovery of partially denervated mouse SGs (Kennedy and Sakuta, 1984; Kennedy et al., 1988). We suggest that the response of sudomotor nerves to neuropathic disease is similar to the well-known time-gated reactions of $\alpha$ motor axons, that is, a decrease in number of axons to the end organ followed by reactive collateral branching with partial reinnervation, remodeling of the terminal innervation, and enlargement of the innervation territory of surviving axons, and finally, eventual uncompensated denervation as the disease progresses. According to this hypothesis, reactive hyperinnervation is possible at an early stage and could account for the locally exaggerated indirect sweat response found at proximal sites during iontophoresis of acetylcholine in some diabetic subjects (Low et al., 1983; Levy et al., 1992) and for the unusually large sweat droplets secreted from some SGs (Kihara et al., 1993). The immunohistochemical demonstration of hyperinnervation in the skin of rats recently made diabetic would support this idea (Karanth et al., 1990). 

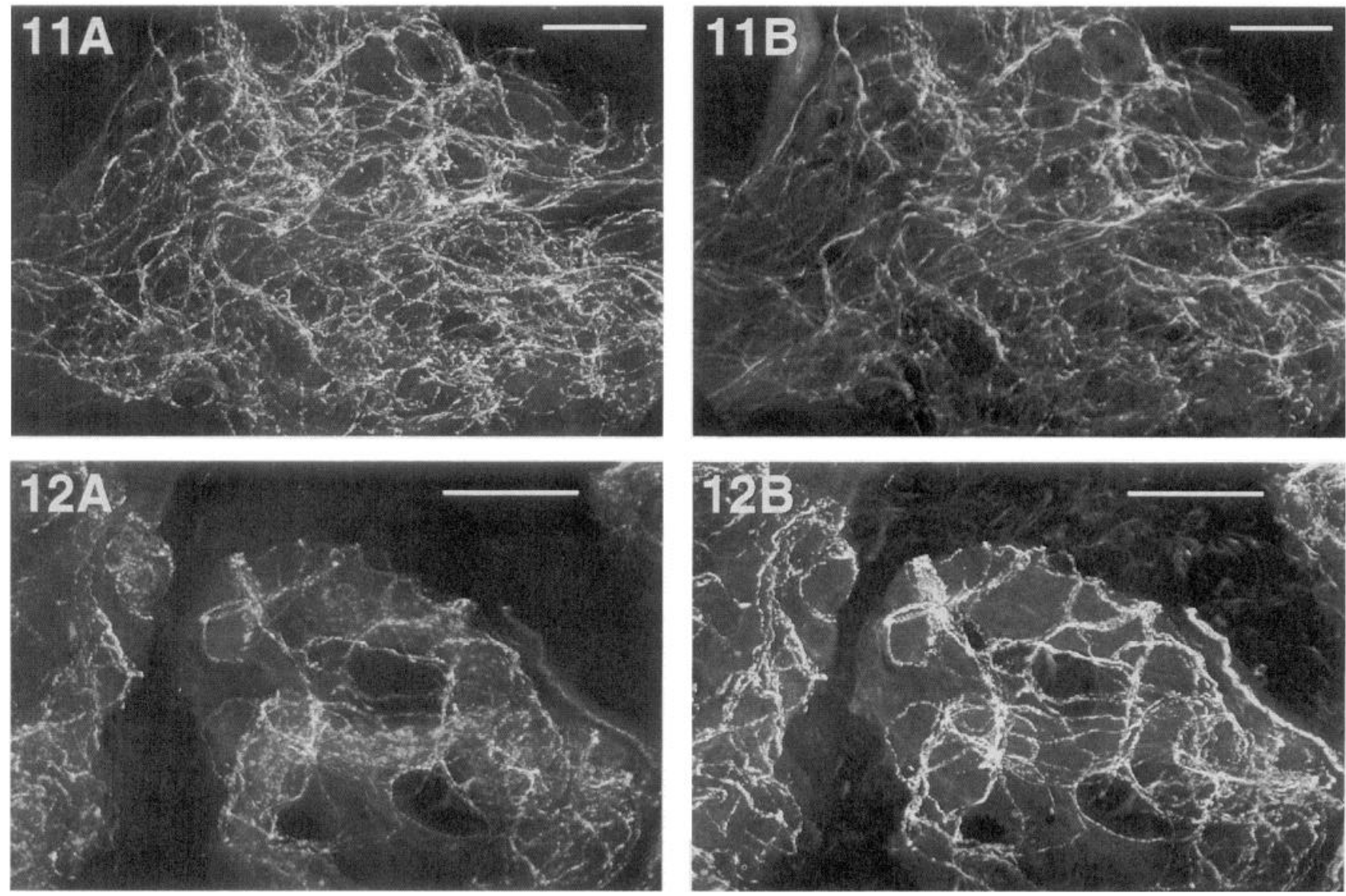

Figure 11. The interrelationship of m-PGP 9.5-ir and VIP-ir in the SG. This section was double stained with polyclonal rabbit anti-VIP and cyanine 3.18-labeled goat anti-rabbit IgG, and then monoclonal mouse anti-PGP 9.5 and cyanine 5.18-labeled goat anti-mouse IgG. The micrographs are projections of three optical sections collected at $2 \mu \mathrm{m}$ intervals with a $20 \times$ Nikon Fluor objective. $A$, VIP-containing axons encircle the sweat tubule and are present in large numbers. B, M-PGP 9.5-containing axons occur in the same pattern as the VIP-containing axons. These antigens almost completely coexist in SGs. Scale bars, $100 \mu \mathrm{m}$.

Figure 12. The interrelationship of m-PGP 9.5-ir and Synap-ir in the SG. This section was double stained with polyclonal rabbit anti-Synap and cyanine 3.18-labeled goat anti-rabbit IgG, and then monoclonal mouse anti-PGP 9.5 with cyanine 5.18-labeled goat anti-mouse IgG. The micrographs are projections of five optical sections collected at $2 \mu \mathrm{m}$ intervals with a $20 \times$ Nikon Fluor objective. $A$, Synap localization is present in axons that encircle the SG tubules. The pattern is characteristic of the innervation seen with other neuropeptides. $B$, M-PGP 9.5 immunoreactivity is present in essentially the same distribution as seen for Synap. Scale bars, $100 \mu \mathrm{m}$.

Denervation hypersensitivity is a less likely alternative etiology because that phenomenon is less prevalent in distal, more severely denervated skin (Levy et al., 1991) and most clinical tests for depressed sudomotor function in neuropathy depend upon the absence of denervation hypersensitivity in sweat glands (Kennedy and Navarro, 1993).

This study does not address the question of whether human eccrine SGs differ according to body location. It is generally recognized that there is great variation in the number of SGs in different body areas. We have observed that the size and shape of SGs are also variable; SGs deeper in the dermis of glabrous skin of the extremities and head are rounded whereas SGs situated between hair follicles are elongated along the axis of the follicle. Furthermore, there are functional differences in the recruitment order of SGs on the finger pulp, a location where individual glands can be identified and retested at long-time intervals (Kennedy et al., 1987). At the onset of sweating some identifiable SGs are always the first recruited. First-recruited glands have the largest sweat droplets; later-recruited SGs have progressively smaller droplets. Sato and Sato (1983) showed that sweat tubules of human SGs vary in length and diameter; larger SGs have a higher sweat rate per gland, a higher sweat rate per unit tubular length, and an increase in sensitivity to methacholine. Combining recruitment information with Sato's findings suggests that droplet size and perhaps recruitment order are locally determined by sweat tubule size, although recruitment order could also be under central control. We have not found evidence that the configuration of the sweat tubules, the innervation pattern or immunoreactivity of sudomotor nerves varies with body location. We have not investigated whether axon concentration per tubule area is uniform throughout the body.

This study demonstrates the normal innervation of SGs of normal subjects. The eventual goal is to establish an information base of the total innervation of normal human skin that is useful for diagnosis and follow-up of patients with peripheral nerve disorders. To accomplish this objective, the innervation of hair follicles, arrector pilorum muscles, epidermis, and dermis from different body sites from subjects of various ages must be studied, and methods devised to quantitate the innervation. It is our experience that preparation of tissue sections by immuno- 
histochemical methods for examination with the LSCM and computer quantitation of nerves stained by reaction with PGP 9.5 antibody have the greatest potential to accomplish this goal. In agreement with Karanth et al. (1991) and Dalsgaard et al. (1989a), we found that PGP 9.5 antibodies labeled a larger number of sensory and autonomic nerve fibers more clearly than other nerve markers. PGP 9.5 is a cytoplasmic protein with characteristics of a general neuronal marker that is capable of identifying most cholinergic, adrenergic, and peptidergic neurons (Gulbenkain et al., 1987; Wilson et al., 1988; Dalsgaard et al., 1989a) in several spccics (Wilson ct al., 1988; Karanth et al., 1991). These features are of major importance for detecting abnormal innervation patterns in reaction to disease processes and for obtaining the degree of staining clarity and intensity necessary for computerized quantitation (Levy et al., 1989, 1992; Tenenghi et al., 1991; Kennedy et al., 1993).

\section{References}

Brelje TC, Wessendorf M, Sorenson RL (1993) Multicolor laser scanning confocal immunofluorescence microscopy: practical application and limitations. In: Methods in cell biology, Vol 38 (Matsumoto B, ed), pp 97-181. San Diego: Academic.

Dalsgaard C-J, Jernbeck J, Stains W, Kjartansson J, Haegerstrand A, Ilokfelt T, Brodin E, Cuello AC, Brown JC (1989a) Calcitonin generelated peptide-like immunoreactivity in nerve fibers in the human skin. Histochemistry 91:35-38.

Dalsgaard C-J, Rydh M, Haegerstrand A (1989b) Cutaneus innervation in man visualized with protein gene product 9.5 (PGP 9.5) antibodies. Histochemistry 92:385-390.

Gulbenkian S, Wharton J, Polak JM (1987) The visualization of cardiovascular innervation in the guinea-pig using an antiserum to protein gene product 9.5 (PGP 9.5). J Auton Nerv Syst 18:235-247.

Holthofer H, Virtanen I, Kariniemi AL, Hormia M, Linder E, Miettinen A (1982) Ulex europaeus I lectin as a marker for vascular endothelium in human tissues. Lab Invest 47:60-66.

Jahn R, Schiebler W, Ouimet C, Greengard P (1985) A 38,000-dalton membrane protein (P38) present in synaptic vesicles. Proc Natl Acad Sci USA 82:4137-4141.

Karanth SS, Springall DR, Lucas S, Levy D, Ashby P, Levene MM, Polak JM (1989) Changes in nerves and neuropeptides in skin from 100 leprosy patients investigated by immunocytochemistry. J Pathol 157:15-26.

Karanth SS, Springall DR, Francavilla S, Mirrlees DJ, Polak JM (1990) Early increase in CGRP- and VIP-immunoreactive nerves in the skin of streptozotocin-induced diabetic rats. Histochemistry 94:659-666.

Karanth SS, Springall DR, Kuhn DM, Levene MM, Polak JM (1991) An immunocytochemical study of cutaneus innervation and the distribution of neuropeptides and protein gene product 9.5 in man and commonly employed laboratory animals. Am J Anat 191:369-383.

Kennedy WR, Crabb-Wendelschafer G (1993) The innervation of human epidermis. J Neurol Sci 115:184-190.

Kennedy WR, Navarro X (1993) Evaluation of sudomotor function by sweat imprint methods. In: Clinical autonomic disorders: evaluation and management (Low PA, ed). Boston: Little, Brown.

Kennedy WR, Sakuta M (1984) Collateral reinnervation of sweat glands. Ann Neurol 15:73-78.

Kennedy WR, Sakuta M, Quick DC (1984a) Rodent eccrine sweat glands: a case of multiple efferent innervation. Neuroscience 11:741749.

Kennedy WR, Sakuta M, Sutherland D, Goetz FC (1984b) Quantitation of the sweating deficiency in diabetes mellitus. Ann Neurol 15: $482-488$.
Kennedy WR, Navarro X, Grampie M, Quick DC, Sakuta M (1987) Orderly recruitment of sweat gland secretion. Ann Neurol 22:150.

Kennedy WR, Navarro X, Kamei H (1988) Reinnervation of sweat glands in the mouse: axonal regeneration versus collateral sprouting. Muscle Nerve 11:603-609.

Kennedy WR, Crabb-Wendelschafer G, Ruppman DJ, Carpenter C, Brelje TC (1993) A quantitative study of nerves in sweat glands in human and diabetic subjects. Ann Neurol 34:269-270.

Kihara M, Opfer-Gehrking TL, Low PA (1993) Comparison of directly stimulated with axon-reflex-mediated sudomotor responses in human subjects and in patients with diabetes. Muscle Nerve 16:655-660.

Landis SC, Fredieu JR (1986) Coexistence of calcitonin gene-related peptide and vasoactive intestinal peptide in cholinergic sympathetic innervation of rat sweat glands. Brain Res 377:177-181.

Levy DM, Karanth SS, Springall DR, Polak JM (1989) Depletion of cutaneus nerves and neuropeptides in diabetes mellitus: an immunocytochemical study. Diabetologia 32:427-433.

Levy DM, Reid G, Abraham RR, Rowley DA (1991) Assessment of basal and stimulated sweating in diabetes using a direct-reading computerized sudorometer. Diabetic Med 8:S78-S81.

Levy DM, Tenenghi G, Gu X-H, Abraham RR, Springall DR, Polak JM (1992) Immunohistochemical measurements of nerves and neuropeptides in diabetic skin: relationship to tests of neurological function. Diahetologia 35:889-897.

Lindh B, Lundberg JM, Hokfelt T, Elfvin L-G, Fahrenkrug J, Fischer J (1987) Coexistence of CGRP- and VIP-like immunoreactivities in a population of neurons in the cat stellate ganglia. Acta Physiol Scand 131:475-476.

Lindh B, Haegerstrand A, Lundberg JM, Hokfelt T, Fahrenkrug J, Cuello AC, Graft J, Massoulie J (1988) Substance P-, VIP- and CGRPlike immunoreactivities coexist in a population of cholinergic postganglionic sympathetic nerves innervating sweat glands in the cat. Acta Physiol Scand 134:569-570.

Low PA, Caskey PE, Tuck RR, Fealey RD, Dyck PJ (1983) Quantitative sudomotor axon reflex test in normal and neuropathic subjects. Ann Neurol 14:573-580.

Lundberg JM, Hokfelt T, Schultzberg M, Uvnas-Wallensten K, Kohler C, Said SI (1979) Occurrence of vasoactive intestinal polypeptide (VIP)-like immunoreactivity in certain cholinergic neurons of the cat: evidence from combined immunohistochemistry and acetylcholinesterase staining. Neuroscience 4:1539-1559.

Quick DC, Kennedy WR, Yoon KS (1984) Ultrastructure of the secretory epithelium, nerve fibers and capillaries in the mouse sweat gland. Anat Rec 208:491-499.

Sato K, Sato F (1983) Individual variations in structure and function of human eccrine sweat gland. Am J Physiol 245:R203-R208.

Tenenghi G, Bunker CB, Yu-Fei L, Springall DR, Cowen T, Dowd PM, Olak JM (1991) Image analysis quantification of peptide-immunoreactive nerves in the skin of patients with Raynaud's phenomenon and systemic sclerosis. J Pathol 164:245-252.

Thompson RJ, Day INH (1988) Protein gene product (PGP) 9.5-a new neuronal and neuroendocrine marker. In: Neurobiological research, Vol 2, Neuronal and glial proteins: structure, function and clinical application (Marangos PJ, Campbell IC, Cohen RM, eds), pp 209-228. San Diego: Academic.

Vaalasti A, Tainio H, Rechardt L (1985) Vasoactive intestinal polypeptide (VIP)-like immunoreactivity in the nerves of human axillary sweat glands. J Invest Dermatol 85:246-248.

Wilson POG, Barber PC, Hamid QA, Power BF, Dhillon AP, Rode J, Day INM, Thompson RJ, Polak JM (1988) The Immunolocalization of protein gene product 9.5 using rabbit polyclonal and mouse monoclonal antibodies. Br J Exp Pathol 69:91-104.

Yoneda K, Yanagihara M, Mori S (1991) Immunohistochemical study of human eccrine sweat ducts with anti-keratin antibodies. Am J Dermatopathol 13:475-480.

Zamboni L, De Martino C (1967) Buffered picric acid-formaldehyde: a new rapid fixative for electron microscopy. J Cell Biol 35:148A. 